\title{
EARLY HISTORY OF GEOMETRIC PROBABILITY AND STEREOLOGY
}

\author{
Magdalena HyKŠOvá凶,1, Anna Kalousová² And IVAn SAXL ${ }^{\dagger, 3}$ \\ ${ }^{1}$ Institute of Applied Mathematics, Faculty of Transportation Sciences, Czech Technical University in Prague, \\ Na Florenci 25, CZ-110 00 Praha 1, Czech Republic; ${ }^{2}$ Department of Mathematics, Faculty of Electrical \\ Engineering, Czech Technical University in Prague, Technická 2, CZ-166 27 Praha 6, Czech Republic; ${ }^{3}$ In \\ memoriam \\ e-mail: hyksova@fd.cvut.cz, kalous@math.feld.cvut.cz \\ (Received October 27, 2011; revised January 2, 2012; accepted January 2, 2012)
}

\begin{abstract}
This paper provides an account of the history of geometric probability and stereology from the time of Newton to the early $20^{\text {th }}$ century. It depicts the development of two parallel paths. On the one hand, the theory of geometric probability was formed with minor attention paid to applications other than those concerning spatial chance games. On the other hand, practical rules for the estimation of area or volume fraction and other characteristics, easily deducible from the geometric probability theory, were proposed without knowledge of this branch. Special attention is paid to the paper of J.-É. Barbier, published in 1860, which contained the fundamental stereological formulas, but remained almost unnoticed by both mathematicians and practitioners.
\end{abstract}

Keywords: geometric probability, history, stereology.

\section{INTRODUCTION}

The first known problem related to geometric probability can be found in a private manuscript of Isaac Newton (1643-1727) previously written between the years 1664-1666, but not published until the $20^{\text {th }}$ century (Newton, 1967). It consists of calculating the chance of hitting one of two unequal areas of a circle by a ball (of negligible size):

If ye Proportion of chances for any stake bee irrational, the interest may bee found after ye same manner. As if ye Radij ab, ac, divide ye horizontal circle bcd in two pts abec \& abed in such proportion as 2 to $\sqrt{5}$. And if a ball falling perpendicularly upon ye center a doth tumble into ye portion abec I win (a): but if ye other portion, I win b, my hopes is worth $(2 a+\sqrt{5} b) /(2+\sqrt{5})$.

... if a die bee not a regular body but a Parallelepipedon or otherwise unequall sided, it may be found how much one cast is more easily gotten than other. (Newton, 1967, p. 60-61)

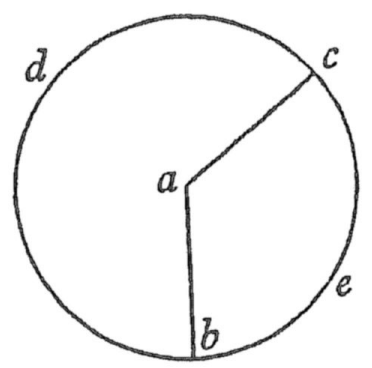

Fig. 1. Newton's illustration (Newton, 1967).
Newton wrote his note after reading the treatise (Huygens, 1657) to point out that probability can be irrational. What is even more remarkable is his claim that chance is proportional to area fraction and the proposal of a frequency experiment for chance estimation. More generally, applying Newton's idea to the ball of negligible size falling $N$ times on an area $C=A \cup B$ and hitting $n$ times the region $A$, and setting $a=1, b=0$, Newton's hope would be $n / N$, which estimates the area fraction of $A$ with respect to $C$. Thus, the first 2D stereological formula, $A_{A}=P_{P}$, was born. In other words, Newton's ideas immediately lead to replacing the counting of events with their measure and to estimating an area fraction by a point count. We can therefore say that Newton also invented stereology.

In a published form, some ideas of geometric probability were applied by Edmond Halley (16561742) in a paper that is generally appreciated as having laid the foundations of a correct theory of life annuities (Halley, 1693). Halley deduced formulas for various annuities first analytically, and then gave their geometric illustration. One year earlier, John Arbuthnot (1667-1735) published the first English work on probability (Arbuthnot, 1692), which contained the translation of Huygens (1657) and some other problems concerning various chance games. Among them we can find an unsolved problem of a completely different nature, which was solved half a century later by means of geometric probability: In a Parallelopipedon, whose Sides are to one another in the Ratio of $a, b, c$ : to find at how many Throws any one may undertake that any given Plane, viz. ab, may 
arise. ${ }^{1}$ The problem was solved by Thomas Simpson (1710-1761) who considered a sphere described round the parallelepiped and imagined a radius passing round the boundary of the given plane. He said that the chance of the given plane being uppermost in a single throw was equal to the ratio of the spherical surface bounded by the moving radius to the whole sphere (Simpson, 1740). In this way, he reduced the problem to finding the area of a certain portion of the surface of a sphere and thus anticipated the measure of the bundle of lines in space passing through a given point.

\section{BUFFON'S PROBLEMS}

Geometric probability is inseparably connected with the name of Georges-Louis Leclerc, later Comte de Buffon (1707-1788), who is generally regarded to be the founder of the discipline. Fontenelle (1735, pp. 43-35) reports about Buffon's presentation of the solutions of franc-carreau and needle problems at the French Royal Academy of Sciences in $1733,{ }^{2}$ and some generalizations can be found in Buffon (1777). ${ }^{3}$ Recall that in the franc-carreau game, popular at the French court, a round coin is tossed at random on a large plane area covered by regular tiles (in the 1733 presentation, Buffon considered squares; the 1777 paper also dealt with triangles, rhombi and hexagons); one of the players bets that the coin hits only one tile while the other bets that it hits more of them. Finding the odds of both players in this game is clearly similar to the above described Newton's problem: each player needs the coin centre to hit a certain subset of a given set. But the needle problem is a completely different and new one - here an interaction of two 1D sets in 2D is examined: ${ }^{4}$

In a room, the floor of which is simply divided by parallel joints, a rod is thrown in the air, and one of the players bets that the rod will not cross any of the lines while the other bets that it will cross several of them. The odds for these two players are required. We can play this game on a board with a sewing needle or a pin without a head. (Buffon, 1777, pp. 100-101)

Denote by $l$ the rod (needle) length and by $d>l$ the distance of unbounded parallels. Using integral calculus, Buffon obtained the odds $\left(1-\frac{2 l}{\pi d}\right):\left(\frac{2 l}{\pi d}\right)$ and used them to calculate the ratio $l / d$ ensuring a fair game for players betting on hits or misses; obviously, the odds are equal for $l / d=\pi / 4$. Buffon (1777) also considered a generalization where the rod was thrown down on a square grid, but he gave an incorrect result.

The needle problem deserves several comments concerning its relation to stereology. The determined odds correspond to the hitting probability

$$
P=\frac{2 l}{\pi d} \text {. }
$$

Noting that $1 / d$ is the length intensity $L_{A}$ of the system of parallels $\mathscr{T}$, and estimating $P$ by the relative frequency $N / n$, where $n$ is the number of randomly thrown needles $\mathscr{L}$ of total length $L=n l$, and $N$ the number of successful hits $\mathscr{L} \uparrow \mathscr{T}$, Buffon's result can be rewritten as

$$
L=\frac{\pi}{2} \cdot \frac{\bar{N}}{L_{A}}
$$

which is the equation for estimating the length of a curve (Steinhaus, 1930b), but in the present case it relates to a compact random segment (fibre) set.

Besides sections, projections are also important tools of geometric sampling and subsequent inference (Davy and Miles, 1977). Moreover, by the Hadwiger characterization theorem (Hadwiger, 1957), the set of all possible mean projection measures together with the Lebesgue measure and Euler-Poincaré characteristics form the basis for the $(m+1)$ dimensional vector space of all convex-continuous motion invariant valuations defined on the convex ring in $\mathscr{R}^{m}$ (Klain and Rota, 1997). By the Cauchy-Crofton formula (Eq. 7) for convex bodies in 2D the relation $L=\pi \bar{w}$ holds between the length of the perimeter $L$ and the mean projection (also called the mean width) $\bar{w}$. Consequently, the hitting probability (Eq. 13) can be written as $P=\bar{w} / d$, which is just the way the formula was derived for the needle, namely by calculating its mean projection into the normal of parallels $\mathscr{L}$ (note that the perimeter of a $1 \mathrm{D}$ segment embedded in $\mathscr{R}^{2}$ equals twice its length). Hence if such a relation was valid in general for convex figures, it would be possible to estimate the mean width $\bar{w}$ or the perimeter $L$ of arbitrary figures by their repeated throwing on the test

\footnotetext{
${ }^{1}$ Quotation from (Simpson, 1740, p. 67); the original formulation in Arbuthnot (1692) was in Latin. Arbuthnot wrote that he left the Solution to those who think it merits their pains (Todhunter, 1865, p. 53).

${ }^{2}$ The franc-carreau with square tiles was already described in the letter written by Gabriel Cramer to James Stirling on February 22, 1732, published in (Tweedie, 1922, pp. 122-128). Cramer remarked that its solution for a round coin was not difficult, but for a square one, he was not able to solve it. On the other hand, Buffon wrote the first letter to Cramer in 1727 and in 1731 he visited him in Geneva.

${ }^{3}$ The first pages of many of works discussed in this paper, including Buffon (1777), are reproduced in Miles and Serra (1978).

${ }^{4}$ Similarly to Cramer, Buffon mentioned already in the 1733 presentation that the franc-carreau problem would be more complicated if the round coin was replaced e.g., by a square one (or a Spanish pistol). Since he did not know how to solve it, he considered only a wand; to simplify the problem further, he started with parallels and only in the later paper he tried to generalize the solution to the square grid. Let us further remark that Buffon (1777) also considered more players betting on hitting different numbers of commissures.
} 
lines (or equivalently by laying on them repeatedly an isotropic and uniform random test system $\mathscr{L}$ ) and counting the fraction of successful hits.

Buffon's problem remained unnoticed by his contemporaries. It received considerable attention only after Pierre-Simon de Laplace (1749-1827) presented - without any reference to its author - both the needle problem and its generalization with two systems of parallels forming a rectangular (not only squared) grid with correct solutions. Laplace (1812) introduced the discussion of these problems with the remark that probability theory could also be used for curve rectification and surface quadrature. Nevertheless, he gave only one example, namely the estimation of the perimeter $2 \pi$ of the unit circle, based on repeatedly throwing a narrow cylinder on a system of parallels. Unfortunately, since Todhunter (1865), Laplace's result was often referred to as the application of Buffon's needle problem to the estimation of $\pi$ (in school mathematics, it is usually the only "application" of the needle problem until today).

Since then, other mathematicians introduced some generalizations. For example, Isaac Todhunter (1820 1884), a lecturer at St John's College, Cambridge, who popularized geometric probability by several exercises in his textbook on integral calculus, considered a cube, a rod of the length equal to the multiple of $d$, and an ellipse to be thrown on the plane ruled with equidistant parallels at a distance $d$ (Todhunter, 1857), later also a closed curve without singular points with the greatest diameter ${ }^{5}$ less than $d$ (1862 edition of the same book). With the reference to the earlier presentation in Fontenelle (1735), Buffon's problems were also analysed in a historical overview (Todhunter, 1865), where a simple derivation of a correct hitting probability for a rectangular grid was given as well. While Laplace (1812) divided rectangles into particular parts and for each of them he "measured" the favourite positions of a needle, Todhunter (1865) used a unique integral over an angle formed by the needle and one of the parallel systems.

Gabriel Lamé (1795-1870) included Buffon's needle problem and its generalizations to a circle, an ellipse and regular polygons in his lectures held at the Sorbonne. Inspired by these lectures, Joseph-Émile Barbier (1839-1889) published a general theorem concerning the mean number of intersections of an arbitrary curve with a system of parallels and, what is remarkable, he replaced equidistant parallels by an arbitrary system of lines or even a unique curve of constant length per unit area, and came to the estimator Eq. 16. Moreover, he extended his results to $3 \mathrm{D}$ and formulated three more theorems that express other contemporary fundamental stereological formulas (Eqs. 17-19) for surface area and curve length estimation. Unfortunately, his contribution remained unknown for some time to Todhunter as well as to Crofton, and its immediate stereological applications were not appreciated until the beginning of the $21^{\text {st }}$ century - see Kalousová (2009). The section after next will therefore describe Barbier's contribution in more detail.

\section{AUGUSTIN-LOUIS CAUCHY}

A.-L. Cauchy (1789-1857) did not speak about "geometric probability", but he derived several theorems with interesting practical implications for length and surface area estimation which were later rediscovered or reformulated in the terms of geometric probability. The lithograph (Cauchy, 1832) starts with the theorem stating that the equality ${ }^{6}$

$$
L=\frac{1}{4} \int_{-\pi}^{\pi} W(\theta) \mathrm{d} \theta
$$

holds for the length $L$ of a system $\mathscr{L}$ of arbitrary curves, where $W(\theta)$ denotes the length of the total [orthogonal linear] projection of $\mathscr{L}$ onto a straight line forming an angle $\theta$ with a fixed axis (each subinterval of the projection is counted as many times as there are points on the curve which project onto it).

Cauchy gives a brief direct demonstration and then shows that Eq. 3 can also be derived from another theorem stating that $L$ can be approximated by

$$
L \approx \frac{\pi}{2} \cdot \overline{W_{n}}
$$

where $\overline{W_{n}}$ denotes the mean value of the total projection of $\mathscr{L}$ onto $n$ radial lines separated by equal angles, and the error of this approximation is smaller than $\frac{1}{2} \pi \overline{W_{n}} / n^{2}$. In a detailed demonstration, Cauchy decomposes $\mathscr{L}$ into infinitesimal line segments and for any of them he investigates the projections. Increasing $n$ to infinity, Cauchy obtains (in our notation)

$$
L=\frac{\pi}{2} \cdot \bar{W},
$$

where $\bar{W}$ is the mean value

$$
\bar{W}=\frac{\int_{-\pi}^{\pi} W(\theta) \mathrm{d} \theta}{\int_{-\pi}^{\pi} \mathrm{d} \theta}=\frac{1}{2 \pi} \int_{-\pi}^{\pi} W(\theta) \mathrm{d} \theta,
$$

which implies Eq. 3. In a corollary to the first theorem Cauchy remarks that for a closed convex curve, $W(\theta)$

\footnotetext{
${ }^{5}$ Todhunter used the term diameter in the sense of our width and greatest diameter in the sense of our diameter.

${ }^{6}$ The notation is slightly changed with respect to the following parts of this paper.
} 
is reduced to twice the ["usual" orthogonal linear] projection $w(\theta)$. From today's point of view we can notice that Eq. 3 and Eq. 5 immediately imply the so-called Cauchy-Crofton formula, which enables the rectification of a closed convex curve by finding its mean width $\bar{w}$, i.e., the mean length of its orthogonal projections onto an isotropic bundle of directions (see Fig. 2): ${ }^{7}$

$$
L=\int_{0}^{\pi} w(\theta) \mathrm{d} \theta=\pi \bar{w} .
$$

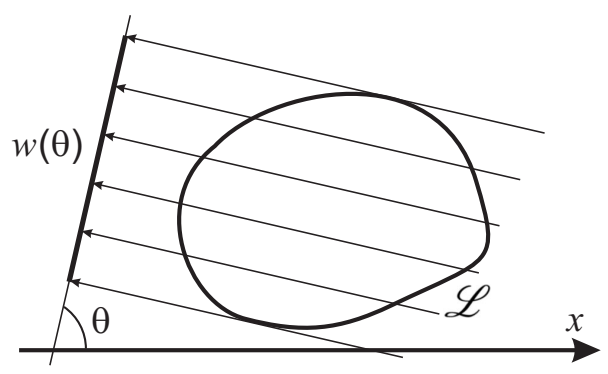

Fig. 2. Illustration of the notation used in Eq. 7.

Cauchy continues into 3D and proposes a practical procedure for surface area estimation: for any surface $\mathscr{S}$ with surface area $S$, he considers its total projections into $n$ planes containing faces of a convex polyhedron that lies between concentric spheres of radii $r, r(1+\varepsilon)$, and shows that $S$ can be approximated by twice the mean area $\overline{A_{n}^{\prime}}$ of these projections,

$$
S \approx 2 \overline{A_{n}^{\prime}}
$$

with an error less than $2 \overline{A_{n}^{\prime}} \cdot\left[(1+\varepsilon)^{2}-1\right]$. Increasing $n$ to infinity, Cauchy replaces a polyhedral face by a differential element of a unit sphere and gets

$$
S=2 \cdot \overline{A^{\prime}},
$$

where $\overline{A^{\prime}}$ is the mean value

$$
\overline{A^{\prime}}=\frac{1}{4 \pi} \int_{-\pi}^{\pi} \int_{0}^{\pi} A^{\prime}(\phi, \theta) \sin \phi \mathrm{d} \phi \mathrm{d} \theta ;
$$

here $A^{\prime}(\phi, \theta)$ denotes the area of the total projection of the surface $\mathscr{S}$ into a plane, the normal of which is given by the co-latitude $\phi$ and the longitude $\theta$ in a fixed coordinate system (see Fig. 3). ${ }^{8}$ Eqs. 9,10 obviously imply the formula for the surface area of $\mathscr{S}$ :

$$
S=\frac{1}{2 \pi} \int_{-\pi}^{\pi} \int_{0}^{\pi} A^{\prime}(\phi, \theta) \sin \phi \mathrm{d} \phi \mathrm{d} \theta .
$$

Cauchy also remarks that Eq. 11 can be demonstrated directly by decomposing $\mathscr{S}$ into infinitesimal planar elements. As in the case of curves, he notes that for a closed convex surface $\mathscr{S}$, the total projection $A^{\prime}(\phi, \theta)$ is reduced to twice the ["usual" orthogonal planar] projection. Thus the well-known formula for convex bodies,

$$
S=4 \cdot \bar{A},
$$

where $\bar{A}$ denotes the mean projected area of $\mathscr{S}$ (taken over all possible orientations in space), again immediately follows from the theorems derived by Cauchy already in 1832 .

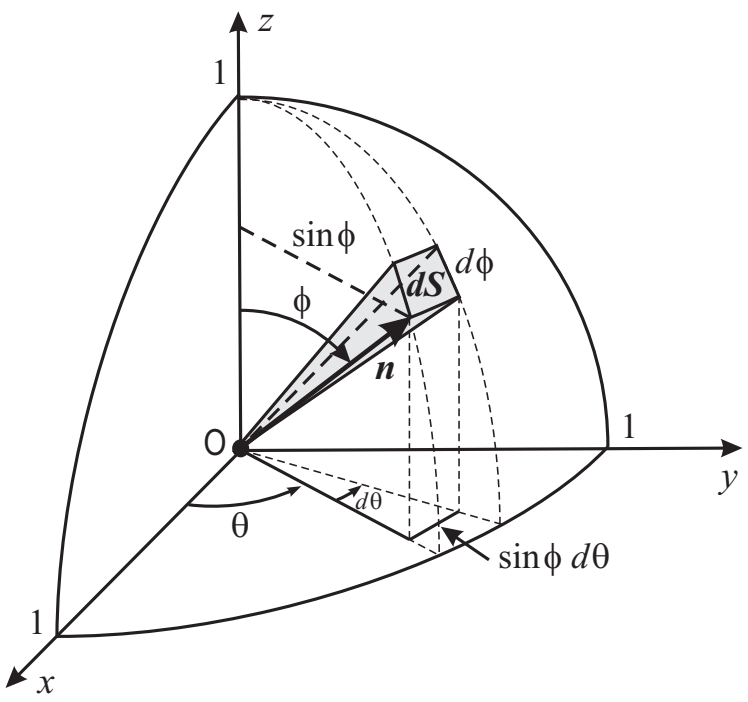

Fig. 3. Illustration of the notation used in Eq. 10.

Cauchy was aware of the importance of his results for the rectification of curves and the quadrature of surfaces, although he gave only several examples concerning circle, ellipse, sphere and ellipsoid. Unfortunately, the lithograph (Cauchy, 1832) was not easily accessible to a wide audience and even though it was reprinted in Mémoires de l'Académie des Sciences in 1850, many authors later referred only to the communication (Cauchy, 1841) published in Comptes Rendus, where the theorems providing Eqs. 3,11 are merely stated and remarks on the approximations (Eqs. 4,8$)$ are missing (although there are some other theorems concerning, e.g., the upper bound of the length or surface area estimates). One of the earliest exceptions was J.-É. Barbier, to whom the next section is devoted.

\section{JOSEPH-ÉMILE BARBIER}

J.-É. Barbier (1839-1889) studied at the École Normale Supérieure, where he attended (among others) mathematical lectures by Joseph Bertrand (1822-1900). Moreover, he attended the mentioned Lamé's lectures at the Sorbonne. He started his career in 1860 as a professor at a Lycée in Nice and

\footnotetext{
${ }^{7}$ Note that for a circle we obtain $L=\pi d$, where $d$ is its diameter; for a square with the side $a$, the mean width is $4 a / \pi$.

${ }^{8}$ The figure is adapted from Czuber (1884a); Cauchy (1832) gave no illustrations.
} 
later he worked as an assistant astronomer at the Observatoire de Paris. In 1865, due to developed mental problems, he left Paris, broke all contacts with colleagues and friends and was not heard from again for the next fifteen years. In 1880, his former lecturer Joseph Bertrand found him at an asylum in Charenton-St-Maurice and encouraged him to return to mathematical research. Two years later Barbier won (thanks to Bertrand's intervention) the Francoeur' prize for an article published in Comptes Rendus (Barbier, 1882). This enabled him to leave hospital and to spend the rest of his life more pleasantly. During the 1880s, he published (with one exception in Comptes Rendus) 14 communications upon various branches of mathematics; three of them (Barbier, 1882; 1887a;b) concerned the probability theory (estimation of $\pi$ based on binomial distribution, a ballot problem etc.) and are referred to and discussed in the recent literature in the context of Barbier's relation to J. Bertrand.

From the stereological point of view, the most important one is the paper that Barbier (1860) wrote during his studies. It starts with the exposition of the needle problem, attributed to Laplace, and its generalizations due to Lamé. Then Barbier turns to a convex disk of arbitrary shape with perimeter $L$ that cannot in any position in the plane intersect more than one dividing parallel, and proves that the probability of hitting some of the lines is

$$
P=\frac{L}{\pi d} .
$$

To prove it, Barbier follows the ideas typical for the analysis of hazards, acknowledging Bertrand's (Bayesian) preference for expectation over probability: consider a convex polygon having $m$ sides of the length $c$ and a diameter less than the parallels distance $d$; evidently, each of the sides has the same chance to cross one of the parallels. Now imagine a game of $m$ players, in which each side belongs to one of them and the fact that a side hits some parallel is connected with certain prize. Before each throw, the "mathematical expectations" of all the players are equal, say $E$. If a player buys $n$ sides, his expected value is therefore $n E$, i.e., proportional to the number of sides, and it is not changed by any deformation maintaining the sides length and convexity of the polygon (so that its boundary has two intersections with any line it crosses) and keeping the diameter less than $d$. Thus the hitting probability (multiplied by the award to give $n E$ ) is also proportional to the number of sides. Approximating any convex disk with the perimeter $L$ and a diameter less than $d$ by such a deformed polygon, the hitting probability Eq. 13 follows immediately from Lamé's result for regular polygons.

It is worth noting that Barbier showed that Eq. 13 can also be derived directly from the aforementioned considerations: since the hitting probability is equal for all convex figures with the same perimeter $L$ and diameter less than $d$, it is sufficient to consider the simplest case, namely a circle with radius $r$ where $L=$ $2 \pi r, r<d / 2$. Such a circle hits some of the parallels if the distance between its centre and the parallel is less than $r$. Thus the hitting probability is

$$
P=\frac{2 r}{d}=\frac{2 \pi r}{\pi d}=\frac{L}{\pi d} .
$$

As Barbier also remarks, a slim rod (needle) of length $l$ can be regarded as the limit of an ellipse with zero minor axis and perimeter $L=2 l$, which immediately implies Eq. 1.

For a non-convex disk, Barbier shows that the hitting probability is equal to the hitting probability of its convex hull; it is therefore sufficient to restrict the investigations to convex figures. If the diameter of the thrown convex figure is greater than the distance $d$, Barbier considers (in our notation) its projection $w(\boldsymbol{\theta})$ onto a straight line forming an angle $\theta$ with a fixed direction, denotes $w_{r}(\boldsymbol{\theta})=\min (w(\boldsymbol{\theta}), d)$ and states:

$$
P=\frac{1}{\pi d} \int_{0}^{\pi} w_{r}(\theta) \mathrm{d} \theta .
$$

If the diameter does not exceed $d$, Barbier refers to (Cauchy, 1832; 1841) and points out that Eq. 13 follows directly from Eq. 14 and Cauchy's formula (Eq. 3), where the symmetry $W(\theta+\pi)=W(\theta)$ for all $\theta \in[0, \pi]$ is used and $W(\theta)$ is replaced by $2 w(\theta)$.

In the next part of his paper, Barbier turns to the computation of the number of intersections and presents several remarkable results. First, he considers a flexible fibre of an arbitrary length. As before, he replaces it with a broken line of length $L$ consisting of $n$ line segments of the same length $l<d$. Using Eq. 1, the mean number of intersections $\bar{N}=n P$ equals ${ }^{9}$

$$
\bar{N}=\frac{2 L}{\pi d},
$$

which allows the estimation of $L$ by counting the number of intersection points. Then, a completely new idea appears: Barbier replaces equidistant parallel straight lines with a system of arbitrary lines with the only requirement of a constant length per unit area (in the present terminology, a constant length intensity). Analogously he proceeds in $3 \mathrm{D}$ and computes the mean number of intersections of a fibre with a surface

\footnotetext{
${ }^{9}$ In Barbier's article, the mean number of intersections is $L / \pi d$. We do not know whether it is a misprint or Barbier's mistake; nevertheless, Eq. 16 is correct and it follows from the correct formula.
} 
of a known area intensity, of a surface with a fibre of a known length intensity and the mean intersection length of a surface with a surface of a known area intensity. Barbier summarizes his results into the four following theorems (Barbier, 1860, pp. 279-280).

Theorem 1: A plane contains a flexible fibre of length $L$ metres in each its square metre, taking a variable form, and let another flexible fibre of length $l$ metres be randomly thrown on the plane, then the mean number of intersection points oscillates, independently of the number of trials, around $2 L l / \pi$.

Writing $L_{A}$ instead of $L$, we obtain the formula Eq. 2 (with $l$ instead of $L$ ) that can be used for the estimation of the length $l$. On the other hand, the second fibre can be regarded as a probe; denoting by $N_{L}$ the number of intersections per its unit length, we get the formula for an unbiased estimator of the length intensity $L_{A}$,

$$
\left[L_{A}\right]=\frac{\pi}{2} \cdot N_{L}
$$

Theorem 2: Let us imagine an unbounded space divided into cubes of one metre edge. Let us suppose that every such cube contains $S$ square metres of a cloth (that need not be evolvable into a plane). A fibre of length $l$, which passes randomly through the space, traverses the cloth in $(1 / 2) \cdot$ Sl points on average.

Writing $S_{V}$ instead of $S$ and denoting by $N_{L}$ the number of intersection points per unit length of fibre, we obtain the well-known stereological 3D formula for the area intensity $S_{V}$,

$$
\left[S_{V}\right]=2 \cdot N_{L}
$$

Theorem 3: Each cubic metre of an unbounded space is traversed by a fibre of length L metres. Then a cloth of surface area s square metres is intersected by the fibre in $(1 / 2) \cdot$ Ls points on average.

Writing $L_{V}$ instead of $L$ and denoting by $P_{A}$ the number of intersections per unit area, we obtain the stereological 3D formula for the length intensity $L_{V}$,

$$
\left[L_{V}\right]=2 \cdot P_{A} .
$$

Theorem 4: Suppose finally that each cubic metre of a space contains $S$ square metres of cloth. Then the mean length of the intersection of this cloth with another cloth of s square metres is $(3 \pi / 2) \cdot$ Ss.

There is a small error in the constant again. The correct expression is $(\pi / 4) \cdot S s$. Writing $S_{V}$ instead of $S$ and denoting by $B_{A}$ the length of the intersection per unit area, we obtain

$$
\left[S_{V}\right]=\frac{4}{\pi} \cdot B_{A}
$$

Barbier does not explain how he arrived at these results. He only remarks that they are based on the mean projections of lines and surfaces and he computes these values only for a circle and a sphere. The last part of his paper contains various remarks and applications. For example, Barbier considers figures of constant width and proves that the length of all curves of the same constant width is the same, while areas of surfaces of the same constant width can differ.

As mentioned above, Barbier's results went unnoticed by his contemporaries. Nobody realized their potential practical utilization. The stereological formulas, simply deducible from Barbier's theorems, were rediscovered as late as the mid- $20^{\text {th }}$ century by Sarkis Andreevich Saltykov (1945; 1946; 1950), Sergei Ivanovich Tomkeieff (1945) and others, ${ }^{10}$ and they turned out to be part of the general multidimensional theory (Miles, 1972).

\section{ENGLISH FOUNDATIONS}

Besides the contributions of Isaac Todhunter $(1857 ; 1865)$, various problems concerning geometric probability (also called local probability) were published and discussed in the Educational Times and Mathematical Questions ${ }^{11}$ since the 1860s. One of the most famous was the four-point problem proposed by James Joseph Sylvester (1814-1897), then a professor of mathematics at the Royal Military Academy at Woolwich. It concerned the probability that four points taken at random in an infinite plane or in a bounded

\footnotetext{
${ }^{10}$ For a detailed bibliography see Baddeley and Jensen (2005).

${ }^{11}$ The journal Educational Times was launched in 1847 by the College of Preceptors in London, the national body whose aim was to supervise standards of teaching and teacher training in the public service. For its main audience - teachers and students - it ran a section Mathematical Questions where various problems were formulated with the encouragement to readers to send solutions for subsequent publication; this department attracted a great attention even of mathematicians and it developed in such an extent that in 1864 an offshoot journal Mathematical Questions with their Solutions from the Educational Times (hereafter Math. Questions) was founded, which contained many additional solutions and other papers. For more details, see (Grattan-Guinness, 1992).

${ }^{12}$ Sylvester (1864b) attributed this result to Arthur Cayley; nevertheless, they later considered the argument used for the unbounded area erroneous and inclined to believe that the question did not admit a determinate solution - see Sylvester's note to Problem 1832 in Educational Times 18(1865), p. 166, and the abstract of his communication On a Special Class of Questions on the Theory of Probabilities published in the Report of the $35^{\text {th }}$ Meeting of the British Association for the Advancement of Science 35(1865), pp. 8-9.
} 
convex area form a re-entrant quadrilateral (Sylvester, 1864a). For an unbounded area, Sylvester $(1864 a ; b)^{12}$ came to the value $1 / 4$. Surprisingly, the "proofs" of several other and substantially different values were later published in the fifth volume of Math. Questions in 1866: 1/3 (J. M. Wilson, p. 81), 1/2 (G. C. de Morgan, pp. 109) or $1 / 2$ decreased by some indeterminate value (C. M. Ingleby, pp. 81-82, 108109). The problem was completely solved by Blaschke (1917) more than 30 years later. ${ }^{13}$ But already in the 1860 s, it aroused a vivid discussion on the concepts "random points," "random lines" and "randomness" in general which showed the necessity of building solid foundations of geometric probability, stimulated its further development and significantly exceeded the framework of a didactically oriented journal. ${ }^{14}$

Let us at least mention the contribution of the astronomer Hugh Godfray (1866) who claimed that the discordance arose from different conceptions of a "random point" (e.g., the chance of its falling within any given area is proportional to this area, or it is the intersection of two random lines). Moreover, to illustrate the insufficiency of the definition of the word "random" for geometric objects, he anticipated the famous paradox formulated by Bertrand (1889) and gave three different answers to the question of what a chord in a circle drawn at random meant: first, a chord joining any two points of the circumference, all combinations being equally probable; second, any line with a distance from the centre less than the radius, all such distances being equally probable; and third, any line joining two points of the circle, all lengths less than the diameter being equally probable. Godfray said that it could easily be shown that the average lengths of such chords were different, greatest in the second case, shortest in the third one. Then he continued with the probability of intersection of two random chords and gave the values $1 / 3,1 / 2$ and $(3 \pi-8) /(4 \pi)$ for the first, second and third case, respectively. He also recalled the paper by the actuary Wesley Stoker Barker Woolhouse (1866) who had investigated the probabilities of various amounts of intersections for $n$ random chords and advocated the first approach. In a note to (Godfray, 1866), ${ }^{15}$ Woolhouse claimed that the latter two cases were quite artificial and that the only legitimate and acceptable conception, consistent with our notions of the meaning of a random chord, was the first one. In the next volume of Math. Questions, Godfray (1867) continued with the discussion of the concept of a "random line" and for three different approaches he derived three different probabilities that the length of a line drawn at random from the focus of an ellipse to its perimeter was greater than the semimajor axis. As before, the paper aroused a critique by Woolhouse (1867), according to whom the only fitting conception regarded the chance of the line falling within a given angle with the vertex in the focus to be proportional to the size of this angle.

Woolhouse's note is followed by the paper of another contributor to Math. Questions, William Morgan Crofton (1826-1915), an instructor and, from 1870 , Sylvester's successor in the professorship at Woolwich. Crofton (1867a) gives detailed reasons for the assertion that the only acceptable definition of a "random point" takes the chance of its falling within any given area proportional to this area. For random lines, he considers the equation (see Fig. 4)

$$
x \cos \theta+y \sin \theta=p,
$$

where $p$ and $\theta$ are constants taken at random.

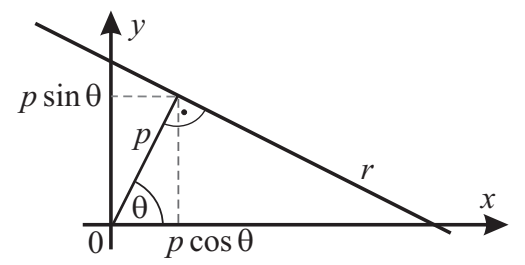

Fig. 4. Illustration of the notation used in Eq. 20.

Crofton argues that this is the only case where each line has the same chance of being selected, contrary to, e.g., the representation by the equation $y=k x+q$ (compare the axis of $x$ and $y$ ). He also returns to the discussion of a random chord in a circle and remarks that the problem lies in the expression "drawing a chord". Provided it means to draw a line taken at random from the random lines that meet the circle, it corresponds to the second case. If it means a random line drawn from a random point on the circumference or a line joining two random points on the circumference, it is the first case. Crofton concludes his note with the following words: The points we are here discussing seem to be the first principles of a new and extensive subject, which offers great difficulties, and calls for much thought and study. At this point, we can speak about the beginning of the systematic development of geometric probability which is no longer a mere tool for solutions of

\footnotetext{
${ }^{13}$ For a bounded area, the solution depends on its shape and has a connection with the rectilinear crossing number of graphs (the minimum number of intersections in any drawing of a graph on $n$ vertices that has straight line segment edges. For a detailed review and the recent state see (Scheinerman and Wilf, 1994) or (Pfiefer, 1989). Let us add that another Sylvester's contribution was the extension of Buffon's needle problem solved by Crofton for two rigidly connected figures to an arbitrarily long chain of such figures (Sylvester, 1890).

${ }^{14}$ This discussion as well as particular contributions is referred to in the monograph (Czuber, 1884b).

${ }^{15}$ Math. Questions 6(1866), p. 81.
} 
various puzzles and games. Crofton (1868) elaborated the topic further and proved several theorems from the future integral geometry. ${ }^{16}$ Several years later he wrote an extensive final chapter On Mean Value and Probability for the second edition of the book of Benjamin Williamson (1877), professor of natural philosophy at the Trinity College, Dublin. It is also worth mentioning Crofton's contribution to the ninth edition of Encyclopaedia Britannica (Crofton, 1885): Its last part titled On local probability begins rather surprisingly with the problem of a variable space $\mathscr{S}$ of mean area $\bar{S}$ included in a space $\mathscr{A}$ of area $A$; the probability that a random point $x \in \mathscr{A}$ hits this variable space $\mathscr{S}$ is $p=\bar{S} / A$. This result just repeats Newton's and Buffon's findings, but the assumption of a variable set was substantially ahead of his time.

For lines in a plane of the form Eq. 20, Crofton introduces the measure by the integral $\iint \mathrm{d} p \mathrm{~d} \theta$ in appropriate limits. He then recalls his previous result, namely that given a convex curve $\mathscr{L}$ of length $L$, the measure of all lines $\mathscr{F}$ hitting $\mathscr{L}$ is ${ }^{17}$

$$
\iint_{\mathscr{F} \uparrow \mathscr{L}} \mathrm{d} p \mathrm{~d} \theta=L
$$

This equation, today called the Crofton formula, can be generalized to higher dimensions and establishes the relation between the measure of an object $M(\mathscr{X})$ and the measure of its sections $\tilde{M}(\mathscr{X} \cap \mathscr{F})$ by flats $\mathscr{F}$ (points, lines, planes etc.). Its immediate consequence is another relation given by Crofton (1885):

$$
\bar{C}=\frac{\iint_{\mathscr{F} \uparrow \mathscr{L}} C \mathrm{~d} p \mathrm{~d} \theta}{\iint_{\mathscr{F} \uparrow \mathscr{L}} \mathrm{d} p \mathrm{~d} \theta}=\frac{\pi A}{L}=\frac{A}{\bar{w}},
$$

where $\bar{C}$ is the mean chord length of a convex region of area $A$ bounded by a curve $\mathscr{L}$ of length $L$, and $\bar{w}$ is the mean width of $\mathscr{L}$ - recall Eq. 7. Also important is the 2D Crofton-Hostinský formula for the third power of chord lengths

$$
\iint_{\mathscr{F} \uparrow \mathscr{L}} C^{3} \mathrm{~d} p \mathrm{~d} \theta=3 A^{2}
$$

from which the estimator $L \overline{C^{3}} / 3$ of the squared area $A^{2}$ immediately follows. Formulae of this type are extremely useful in the stereological analysis of particle aggregates (Miles, 1983).
Crofton (1868) did not investigate the chord lengths yet, but in a remark to Eq. 21 we can find another important stereological result, namely the Cauchy-Crofton formula Eq. $7 .{ }^{18}$ From other problems considered by Crofton (1868), let us at least mention the generalization of Buffon's problem to a "needle" consisting of two rigidly connected figures of diameters not exceeding the distance between parallel lines, later extended by Sylvester (1890) to an arbitrarily long chain of such figures.

Only the 2D problems are solved by Crofton, but once formulated they allow for a generalization to higher dimensions. Crofton can therefore be credited for laying the foundations of geometric probability and for undertaking the first systematic attempt to relate measures of intersections of bodies to their properties. As for the generalization to 3D, Crofton himself outlined it at the end of his 1868 paper; in full detail, it was done by Czuber (1884a;b), whose book Crofton (1885) appreciated in the concluding remark: We have now done enough to give the reader some idea of the subject of local probability. We refer him for further information to the very interesting work just published by Emanuel Czuber of Prague, Geometrische Wahrscheinlichkeiten und Mittelwerte, Leipsic 1884 ...

(Crofton, 1885, p. 788)

\section{EMANUEL CZUBER}

The above-mentioned "very interesting work" (Czuber, 1884a) published by Emanuel Czuber (18511925), at that time a secondary school teacher in Prague, later a professor at the German Technical University in Brno and at the Technical University in Vienna, represented the first monograph summarizing the state of the art of geometric probability of that time and it played an important role in this field for several decades. In 1902 it was translated into French and it remained a classic until Robert Deltheil (1890-1972), a professor at the Toulouse University, published his book (Deltheil, 1926). The first and more extensive part of Czuber's monograph is devoted to geometric probability itself and it provides not only a detailed exposition of results achieved by French and British predecessors, supplemented with historical remarks and many exercises, but also new results and generalizations. For example, Crofton (1868)

\footnotetext{
${ }^{16}$ One of them was the formula $\iint(\alpha-\sin \alpha) \mathrm{d} x \mathrm{~d} y=\frac{1}{2} L^{2}-\pi A$, where $\alpha$ denotes the angle between two tangents from an exterior point $(x, y)$ to the curve of the length $L$ that forms the boundary of a convex region of the area $A$, and the integral is taken over the whole plane outside the boundary. This result and several other relating theorems had already been briefly announced in (Crofton, 1867b). Crofton (1868) appreciated Buffon and Laplace; without mentioning (Barbier, 1860), he remarked that no real attention was devoted to geometric probability till English mathematicians as, e.g., Sylvester and Woolhouse entered this field of research.

${ }^{17}$ Crofton wrote limits only to single integrals; the notation $\mathscr{F} \uparrow \mathscr{L}$ has been added by the authors of this paper for the sake of clarity.

${ }^{18}$ Crofton (1868) gave no reference to Cauchy; the fact that Eq. 7 immediately followed from the theorem stated by Cauchy was pointed out by Czuber (1884a), although the reference concerned only the Comptes rendus report (Cauchy, 1841).
} 
derived key theorems concerning sets of points and straight lines in a plane and briefly outlined a possible generalization to 3D; this generalization was given in full detail by Czuber. Moreover, although the particular results are derived in $2 \mathrm{D}$ and $3 \mathrm{D}$, the introductory chapter contains a definition of geometric probability as a content ratio in $\mathscr{R}^{n}$ :

$$
p=\frac{\iint \ldots \int_{K^{\prime}} \mathrm{d} x_{1} \mathrm{~d} x_{2} \ldots \mathrm{d} x_{n}}{\iint \ldots \int_{K} \mathrm{~d} x_{1} \mathrm{~d} x_{2} \ldots \mathrm{d} x_{n}}
$$

where $K^{\prime} \subseteq K \subset \mathscr{R}^{n}$. Inspired by the discussion in the Educational Times (referred to elsewhere in the book), Czuber recalls that it is often possible to find different solutions of problems concerning geometric probability, and points out that this diversity arises from different conceptions of the random sampling. Thus he also foreshadowed Bertrand's paradoxes.

The second part of the monograph contains the original exposition of the determination of mean values of geometric variables based on geometric probability. As for stereological applications, the book contains only one explicit remark that concerns an experimental rectification of a closed convex curve in 2D. After the proof of the Crofton formula Eq. 21, Czuber recalls another result contained in (Crofton, 1868), namely that the probability that a line hitting a closed convex curve $\mathscr{L}$ of length $L$ hits also a closed convex curve $\ell$ of length $l$ that lies inside $\mathscr{L}$ is $p=l / L$. Then he remarks that this result provides an experimental rectification of a closed convex curve: The curve that has to be rectified is surrounded by another closed convex curve (circle, polygon) of the known length $L$, a great number s of arbitrary straight lines intersecting $L$ are drawn in the plane of both curves, and those intersecting also the curve of the unknown length $l$ are counted; let their number be $m$. The higher $s$, the more accurate the equality $\mathrm{m} / \mathrm{s}=l / L$ holds, which implies $l=\mathrm{Lm} / \mathrm{s}$.

(Czuber, 1884a, p. 116)

We may regret that Czuber does not explicitly mention an analogous surface area estimation. Nevertheless, it immediately follows from his theorem stating that the measure of all lines hitting a closed convex surface $\mathscr{S}$ is proportional by $\pi / 2$ to its surface area $S$. More specifically, in accordance with Crofton's outline, Czuber (1884a) proved:

$$
\int_{0}^{\pi} \int_{0}^{\pi} A(\phi, \theta) \sin \phi \mathrm{d} \phi \mathrm{d} \theta=\frac{\pi}{2} \cdot S,
$$

where $A(\phi, \theta)$ denotes the area of the projection of $\mathscr{S}$ into the plane whose normal has co-latitude $\phi$ and longitude $\theta$ in a fixed coordinate system. In a remark to this theorem Czuber refers to (Cauchy, 1841) where the formula Eq. 11 was stated without any demonstration; probably unaware of the older treatise, Czuber provides the direct proof corresponding to the brief outline mentioned by (Cauchy, 1832) in a note to this formula (see page 4 in this paper) and shows that Eq. 25 can simply be deduced from Eq. 11 .

Some additional original results concerning geometric mean values in $3 \mathrm{D}$ are contained in the paper (Czuber, 1884b). As an example, let us mention the formula for the mean chord length $\bar{C}$ of a convex region of volume $V$, bounded by a closed convex surface $\mathscr{S}$ of surface area $S$ :

$$
V=\frac{1}{4} \cdot S \bar{C}
$$

Czuber returned to geometric probability also in his later publications, e.g., in a comprehensive treatise (Czuber, 1899) devoted to the history of probability theory and its applications, or in the textbook on probability theory and its applications for life insurance (Czuber, 1903); in the second edition of this book published in 1908, the exposition of geometric probability was enriched by the discussion of set theory and its use in probability theory, as it was systematically done by Rudolph Lämmel (1879-1962) in his Ph.D. thesis (Lämmel, 1904).

\section{FURTHER DEVELOPMENT}

Considerable attention to geometric probability was also paid by Joseph Bertrand. In the second volume of (Bertrand, 1864; 1870), he devoted a separate section to Crofton's theorems. Among other results, he returned to the problem of a generalized Buffon's needle consisting of two rigidly connected convex figures of diameters less than the distance of parallels solved by Crofton (1868), and he gave its solution based on Barbier's expectation approach. However, more famous are the so-called Bertrand's paradoxes concerning a random selection from infinite populations (Bertrand, 1889), formulated to warn against a careless use of infinity, ${ }^{19}$ especially his three possibilities of sampling chords in a circle leading to three different answers to the question about the probability that a chord chosen at random is longer than the side $a$ of the inscribed equilateral triangle: ${ }^{20}$ First, one of the chord endpoints is known (by symmetry, this knowledge should not change the outcome) and its direction determined by the angle $\alpha$

\footnotetext{
${ }^{19}$ Bertrand (1889, p. 4) wrote in the introduction: infinity is not a number, it shall not be, without explanation, introduced in the reasoning.

${ }^{20}$ Bertrand $(1889$, p. 4) formulated the problem as follows: A chord is drawn at random in a circle. What is the probability that it is smaller than the side of the inscribed equilateral triangle? But the solution was formulated for chords longer than that side.
} 
from the tangent at the endpoint is chosen at random; the chord is longer than $a$ for $\alpha \in(\pi / 3,2 \pi / 3)$, the required probability is therefore $P_{1}=1 / 3$ (note that the same result arises from the independent choice of chord endpoints with a uniform distribution over the circumference). Second, the chord direction is known and its distance from the circle centre is chosen at random; the chord is longer than $a$ if the distance is less than half of the circle radius $r$, which leads to $P_{2}=1 / 2$. Third, the midpoint is chosen at random; now the chord is longer than $a$ if its midpoint lies inside the concentric circle of the radius $r / 2$, which gives the probability $P_{3}=1 / 4$ equal to the ratio of circle areas. Bertrand (1889, p. 5) then concludes: Among these three answers, which one is right? None of the three is incorrect, none is correct, the question is ill-posed.

We have already mentioned a similar problem formulated by Godfray (1866) and the answer of Woolhouse and Crofton (see p. 7). Bertrand's questions provoked another discussion on the foundations of geometric probability. Henri Poincaré (1854-1912), a professor at the Sorbonne, treated this topic in (Poincaré, 1896); he introduced the concept of probability density, derived its form for the first two cases considered by Bertrand and stated that the problem followed from the fact that they were different. In accord with his conventionalism, Poincaré claims that in general we do not know the nature of the density function, which can be arbitrary, and we must set it at the beginning of our considerations by a meaningful convention. Then he investigates a generalized needle problem. He says that the probability that a given plane figure $\mathscr{F}$ satisfies certain conditions concerning its position is proportional to the integral $\iiint \mathrm{d} x \mathrm{~d} y \mathrm{~d} \omega$, where $(x, y)$ are the Cartesian coordinates of a fixed point $M$ of $\mathscr{F}$ and $\omega$ is the angle between the $x$-axis and a straight line passing through $M$ and rigidly attached to $\mathscr{F}$, since - as he shows - this integral is invariant under rotations and translations. If this convention is adopted and a random chord is regarded as a segment of one of the parallels with the distance $d \leq 2 r$ on which the given circle with the radius $r$ is thrown, then the chord is longer than the side of the inscribed equilateral triangle when the line hits also a concentric circle with the radius $r / 2$; since the probability of this event is equal to the ratio of perimeters of the two circles, we obtain $P_{2}=1 / 2$.

The paradox was later discussed by Czuber (1903; from the second edition published in 1908) who presented three more possibilities, ${ }^{21}$ calculated the corresponding probabilities and pointed out that only the second Bertrand's alternative with $P_{2}=1 / 2$ corresponded to the concept of randomly chosen straight line as it had been introduced by Crofton. Similarly Borel (1909) asserted that the majority of conceivable natural procedures led to $P_{2}$. We have already seen that this solution also corresponds to the motion invariant sampling scheme, which is now generally accepted in geometric probability; nevertheless, this does not answer completely the philosophical background of the problem. Marinoff (1994) argues that Bertrand's answers can be construed as replies to three different questions: the random chord is either generated by a procedure on the circumference of the circle, by a procedure outside the circle, or by a procedure inside the circle (compare again Woolhouse, 1866). He criticises the former literature for the little recognition of the distinction between them and demonstrates that clearly stated variations lead to different, but theoretically and empirically self-consistent solutions. Further, see Plato (1994); Sheynin (1994; 2003), and a study paying attention to Bertrand's teaching and probabilistic thinking (Bru, 2006).

Let us note that independently of Poincaré, the motion invariance requirement was formulated by Élie Cartan (1869-1951), at that time a lecturer at the University at Montpellier, later a professor at the University of Nancy and at the Sorbonne. Cartan (1896) studied multiple integrals over systems of lines in the plane and systems of lines and planes in space and introduced measures of such systems independent (as he proved) of translation and rotation, corresponding to those proposed by Crofton and Czuber, including Eq. 21 and other relations. Nevertheless, there is not a word about geometric probability and its proponents as Cartan was solely dealing with the theory of integrals. In the framework of geometric probability, this topic was investigated by Georg Pólya (1887-1985), a lecturer and later a professor at ETH Zürich. The aim of his paper (Pólya, 1917) was to show (without a reference to Cartan or Poincaré) that the measures of sets of lines and planes on which Crofton and Czuber based the geometric probability theory, were the only legitimate ones, and the reason was again the motion invariance.

At the beginning of the $20^{\text {th }}$ century, interesting contributions to geometric probability were presented by Bohuslav Hostinský (1884-1951), a private associate professor at Charles University in Prague, later a full professor at Masaryk University in Brno. Hostinský (1917; 1920) criticized the traditional

\footnotetext{
${ }^{21}$ One endpoint of the chord is given on the circumference, then another point is chosen inside the circle; two endpoints are independently chosen with uniform distribution over the circle circumference, which is shown to be equivalent with the first possibility with $P_{1}=1 / 3$; two points are independently chosen inside the circle.
} 
solution of Buffon's needle problem for being based on an unrealistic assumption that parallels were drawn on an unbounded board and the probability that the needle midpoint hit a region of a given area was proportional to this area and independent of the position of the region. Hostinský argued that no real experiment could satisfy such an assumption and replaced it by a more realistic one: parallels are drawn on a square table board and the experiment requires the needle to fall on it; now the probability that the needle midpoint hits a square of a given area near the edge of the table is lower than the probability that it hits a square of the same area near the middle. To solve this problem, Hostinský generalizes the method of arbitrary functions introduced by Poincaré $(1896)^{22}$ and supposes that the probability that the needle midpoint falls into a region $M$ inside a square $C$ (the table) is proportional to the integral over $M$ of the form $\iint \varphi(x, y) \mathrm{d} x \mathrm{~d} y$, where $\varphi(x, y)$ is an arbitrary function with continuous partial derivatives in $C$ such that for some constant $K$, the inequalities $\left|\varphi_{x}^{\prime}(x, y)\right|<$ $K,\left|\varphi_{y}^{\prime}(x, y)\right|<K$ hold. In a limiting case where the number of parallels increases to infinity, Hostinský's solution corresponds to the original result of Buffon. Hostinský discussed the French variant of this paper (Hostinský, 1920) in his correspondence with Maurice Fréchet, which could have awoken Fréchet's interest in probability theory (Havlová et al., 2005). Fréchet supplemented Hostinský's ideas in Fréchet (1921); see also Fréchet and Halbwachs (1924). Five years later Hostinský published a French booklet (Hostinský, 1925), in which he extended the contributions of Crofton (1868) and Czuber (1884a;b) for surfaces in space. Besides other results he proved the analogy of the Crofton-Hostinský formula mentioned above, which is probably the reason why Hostinský's name has been associated with the name of Crofton in this connection. For a given closed convex surface $\mathscr{S}$ of surface area $S$ and interior volume $V$ Hostinský proved that the mean value of the fourth power of the chord length $C$ is

$$
\overline{C^{4}}=\frac{12}{\pi} \cdot \frac{V^{2}}{S} .
$$

In the next year, he published the first (and for a long time the sole) Czech book on geometric probability (Hostinský, 1926). Here he proceeds from sets of points over sets of lines in the plane up to sets of lines and planes in space and studies their interactions with curves and surfaces; measures of particular sets are explicitly introduced on the basis of the concept of invariance under translation and rotation. Hostinský also discusses the above-mentioned Bertrand's chord paradox and claims that a reasonable calculation of probability can be carried out only with the respect to experimental conditions under which the choice of a random chord is made. He argues that each possibility is legitimate and each of them corresponds to another experiment. Hostinský's discussion of the influence of experimental realizations on solutions to various problems includes also the generalization of Poincaré's method of arbitrary functions mentioned above.

One year later, Hostinskýs student Josef Bat’a (1894-1929) published a nowadays almost unknown booklet (Bat'a, 1927) where he outlined the history of Bertrand's chord paradox including the contributions of Poincaré and Czuber, derived probability densities for all six possibilities and then reported the results of experiments that he had ran on the basis of Hostinský's proposals for the original three alternatives. In one of the experiments, Bat'a used five transparent discs on which three concentric circles $k, k^{\prime}$ and $k^{\prime \prime}$ with diameters 60,30 and $15 \mathrm{~mm}$ were drawn, and threw them repeatedly (altogether 10,000 times) on a large sheet of paper with two perpendicular systems of parallel lines having the distance $60 \mathrm{~mm}$ ( $k$ hits always some of the lines). For each circle size he recorded the number of intersections with some line at a particular direction and the number of grid points falling inside the circle. He estimated $P_{2}$ by calculating the ratio of the number of lines hitting $k^{\prime}$ (resp. $k^{\prime \prime}$ ) to those hitting $k$ (resp. $k^{\prime}$ ), which gives $\left[P_{2}\right]=0.502$ (resp. $\left[P_{2}\right]=$ $0.4985)$. The ratio of the number of grid points falling into $k^{\prime}$ (resp. $k^{\prime \prime}$ ) to those falling into $k$ (resp. $k^{\prime}$ ) then gives the estimate $\left[P_{3}\right]=0.2426$ (resp. $\left.\left[P_{3}\right]=0.2502\right)$. To estimate $P_{1}$, Bata drew two concentric circles $k, k^{\prime}$ on a sheet of paper and to one point of $k$ he pinned the centre of a transparent circular disc covering $k$, on which a diameter was drawn. He spun the disc and (after some technical improvements) found the ratio of the number of cases when the diameter hit $k^{\prime}$ to all hits of $k$ and obtained the estimate $\left[P_{1}\right]=0.3347$.

The description of the vast development in the following decades exceeds the scope of this paper. Let us only recall the school of Wilhelm Blaschke (1885-1968), a professor of mathematics at the university in Hamburg. Inspired by the lectures of his friend Gustav Herglotz (1881-1953) on geometric probability held in the summer semester 1933 at the university in Göttingen, Blaschke started to lecture on this topic in Hamburg (in the academic year 1934/35 and summer semester 1936), Bulgaria and Romania (in Autumn 1935). Together with his students he also started to publish a series of papers under the generic title integral geometry, denoting by this term the theory based on the concepts of probability, measure, transformation groups and geometry, which

\footnotetext{
${ }^{22}$ Hostinský referred to the second and substantially revised edition of this book from 1912.
} 
he viewed as important as differential geometry; this early research was summarized in the book (Blaschke, 1935). Among Blaschke's students and collaborators belong such well-known personalities as Hugo Hadwiger (1908-1981), later professor at the university in Bern and the author of the book (Hadwiger, 1957) (to name only one publication), Luis Antonio Santaló (1911-2001), later professor at the university in Buenos Aires and the author of the famous monograph (Santaló, 1976), or Shiing-Shen Chern (1911-2004), later professor at the universities in Chicago and Berkeley and the author of various papers that were republished in Cheng et al. (1996). ${ }^{23}$

\section{SOME PRACTICAL PROCEDURES}

In the $19^{\text {th }}$ century, practical procedures were proposed to describe objects scattered in the plane and space, which intuitively and implicitly used the approaches of geometric probability without knowing about its existence. It seems surprising that, for example, the simple point counting method was discovered by geologists almost half a century after the publication of Czuber's monograph on geometric probability, from which it immediately follows. The French geologist and mineralogist Achille Ernest Oscar Joseph Delesse (1817-1881) proposed to estimate the volume fractions of particular minerals in a rock from their area fractions in planar sections. ${ }^{24}$ Although the practical implementation of his area estimation method was rather laborious, consisting in tracing the contours of grains on an oiled paper, colouring the regions with different colours for particular minerals, gluing on a tin foil, cutting, sorting by colours and weighting the particular groups and thus obtaining the searched fractions (Delesse, $1847 \mathrm{a} ; \mathrm{b})$, the idea that the volume estimation does not necessarily require a complete $3 \mathrm{D}$ reconstruction of an object was very important. Various modifications later appeared: e.g., for fine-grained rocks where a microscope was necessary, Henri Clifton Sorby (1856) proposed the use of a camera lucida to draw the exact image of the observed sample, John Joly (1903) replaced mechanical procedure by a graphical area estimation, and Albert Johannsen (1919) proposed the use of a planimeter. In the domain of bioscience, the method of volume estimation by means of measuring areas in parallel plane sections (using a planimeter and magnified images of sections made at definite intervals) was developed and used by William R. Thompson (1932) and his collaborators from the Department of Pathology of Yale University (Thompson et al., 1932). It is worth noting that Thompson (1932) also described the simple counting principle based on section pairs.

The Austrian geologist August Karl Rosiwal (1860-1923) solved the volume estimation problem by examining linear sections (Rosiwal, 1898); in the present notation, he came to the formula $V_{V}=$ $S_{S}=L_{L}$. Rosiwal's method was again very useful in materials science and geology and it simplified the estimation of areas in maps and plans as well. It was further improved, e.g., by Samuel James Shand (1916), who introduced a recording micrometer device that not only performed the measurements but also their addition, and by Ellis Thomson (1930), who transferred the measurement from the microscope to photomicrographs, lantern-slides or on the projection of slides on the blackboard. For the lineal method Thomson used lines forming a square grid drawn on a slide, and for comparison, he also estimated areas directly by counting squares covering the relevant mineral.

With explicit reference to geometric probability, the point counting method was introduced by the Russian geologist, mineralogist and petrologist Andrei Aleksandrovich Glagolev (1894-1969): Imagine that a great number of points are scattered uniformly random in a rock. ... Probability that any of these points falls upon a grain of certain mineral is equal to $p=A / 100$, where $A$ denotes the percentage contents of this mineral in the rock. If we count a high number of points $(n)$ scattered in the rock and the number of points $(m)$ hitting a grain of the given mineral, the ratio $[m / n]$ will be approaching $p$, i.e., the true fraction of the given mineral (Glagolev, 1933, p. 23). In the present notation, we finally have the formula

$$
V_{V}=S_{S}=L_{L}=P_{P} \text {. }
$$

One year later, Glagolev and Gotman (1934) described the application of the point counting method for the

\footnotetext{
${ }^{23}$ After the studies in Beijing, Chern obtained his doctorate in Hamburg as a student of Blaschke and then he spent another year as a post-doctoral fellow in Paris, learning geometry from Élie Cartan. One of his earliest papers deals for example with integral geometry in Klein spaces - see (Chern, 1942).

${ }^{24}$ Delesse considers a rock sample to be placed into a coordinate system and expresses the volume of particular mineral by the integral $\int_{z} f_{i} \mathrm{~d} z$, where $f_{i}$ denotes the area of this mineral in the section by the plane parallel to the plane $x y$. If the composition of the rock is uniform, the mentioned integral expresses the volume of a cylinder with the base of the area $f_{i}$ and height equal to the height of the sample. Volumens of different minerals are then in the same proportions as areas of bases $f_{i}$. Quite naturally, the estimator of the volume of a solid from parallel systematic sections is named after Bonaventura Cavalieri (1598-1647), whose famous principle presented in (Cavalieri, 1635) represents the important step towards a general definition of volume for an arbitrary solid based on the comparison of its lower-dimensional sections with those of a reference solid (for more details on Cavalieri principle, see (Cruz-Orive, 1987)).
} 
exploration of copper ore at the Kounrad deposit. Here samples were taken at one meter intervals throughout the whole depth of a hole and a regular grid of test points was used for their analysis (see Fig. 5).

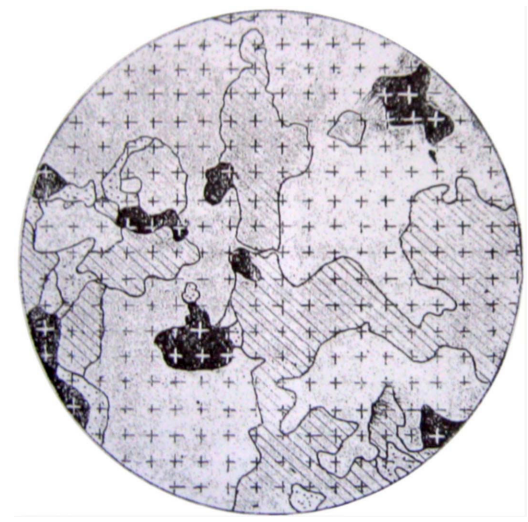

Fig. 5. Illustration from Glagolev and Gotman (1934).

Independently of geologists, the point counting method as a tool for the study of tissue composition was proposed by the physiologist Harold William Chalkley (1943).

At the beginning of the 1930's, a practical method for the estimation of a curve length was proposed by Hugo Dyonizy Steinhaus (1887-1972), a professor at the University in Lwów, later in Wrocław. In (Steinhaus, 1930a) he proved Cauchy's formula Eq. 3 in the form $L=\frac{1}{2} \int_{0}^{\pi} W(\theta) \mathrm{d} \theta$, using parametrization of the curve, some trigonometry and the concept of Riemann's integral. The proof is followed by a remark that it can be generalized to any rectifiable curve by employing the concept of Lebesque integral and that a similar relation also holds for spatial curves projections must only be made on all planes and the mean length of the total projection must be multiplied by $4 / \pi$. However, Steinhaus went even further and proposed to use Eq. 3 as the basis of a new definition of the curve length: we can consider an arbitrary plane set, determine Lebesque measure of its projection onto a straight line (where the segments covered several times should be considered as many times) ... and accept the integral $\frac{1}{2} \int_{0}^{\pi} W(\theta) \mathrm{d} \theta$ as the length of the considered set. ${ }^{25}$ Similarly, Steinhaus claimed that it was advantageous to define the surface area by Eq. 11 .

To obtain an estimate of the curve length, Steinhaus (1930a) used the trapezoid rule for integral approximation. Due to $W(0)=W(\pi)$ he got

$$
L=\frac{1}{2} \int_{0}^{\pi} W(\theta) \mathrm{d} \theta \doteq \frac{\pi}{12} \sum_{i=1}^{6} W\left(\frac{(i-1) \pi}{6}\right),
$$

and described a device constructible as a part of a microscope ocular, which recorded the movement of a groove in a fixed perpendicular direction as its intersection with the curve was moving along the curve length; in this way one of the terms in the sum Eq. 29 was determined and all others were obtained by a successive rotation of the device. ${ }^{26}$ Then Steinhaus proposed a simplified and faster method consisting in covering the given curve by a transparent disc on which two perpendicular systems of parallels with the distance $12 / \pi$ were drawn, counting the number of squares intersected by the curve as if they were passed through by a chess rook (see Fig. 6), and thus obtaining $\frac{12}{\pi}(W(0)+W(\pi / 2))$. After the repetition of the same procedure for two other positions of the disc obtained by the rotation by $\pi / 6$ and $\pi / 3$, the total number $N_{\text {total }}$ of hit squares directly gave the length estimate. Another view on this method is based on the observation that the number of squares calculated in the horizontal direction also represents the number of intersections (for objects with a visible width as rivers on maps, the number of "bridges") of the curve with the system of vertical parallels, similarly for the other direction. Using Eq. 15 with $\bar{N}=N_{\text {total }} / 6$ and $d=12 / \pi$, we get the estimate of $L$ as the total number of intersections with all considered parallels; this procedure was proposed in Steinhaus (1930b).

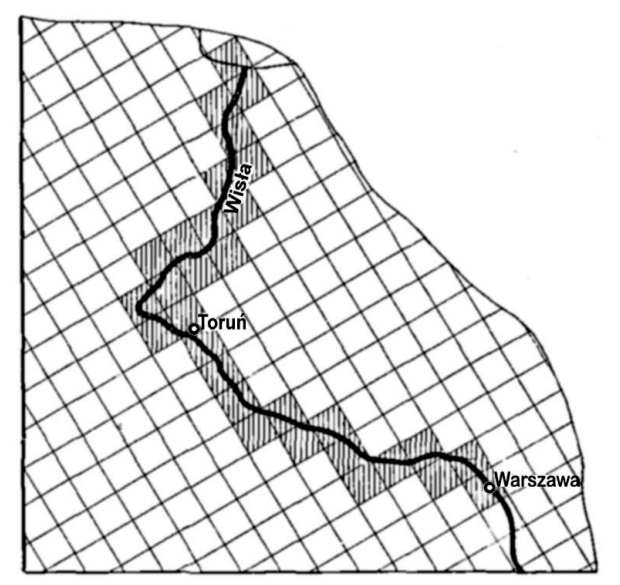

Fig. 6. Illustration from the paper (Steinhaus, 1931).

Steinhaus (1931) presented his method in a journal for geography teachers; using the map of Poland scaled $1: 5000000$, he applied it to the estimation of the length of the river Vistula between Warszaw and the Baltic See (see again Fig. 6). In a complete and

\footnotetext{
${ }^{25}$ (Steinhaus, 1930a, pp. 128-129); Steinhaus used $\alpha$ instead of our $\theta$. He pointed out several advantages of this definition: independence of a parametrization, immediate recognition of the length motion invariance, no need of kinematic concepts of start and end points, applicability to a wider range of curves.

${ }^{26}$ Steinhaus presented this method also in the session of the Polish Mathematical Society on July 3, 1929; in a remark given on October 10, 1929, he appreciated (Cauchy, 1832) - after being notified by B. Hostinský. See Ann Soc Polon Math 9(1930), pp. $192-193$.
} 
accessible way, his length estimation method is also described in (Steinhaus, 1950). ${ }^{27}$

Inspired by Steinhaus' rectification approach, Patrick Alfred Pierce Moran (1917-1988) published the method for the estimation of the surface area of a convex body, based on the identity Eq. 9 and corresponding to Cauchy's outline how to get Eq. 8 see Moran (1944).

At the turn of the $19^{\text {th }}$ and $20^{\text {th }}$ centuries, methods for the determination of the mean grain size as a basic characteristic of technical materials were proposed. They were also based on an intuitive and unfortunately incorrect understanding of the features observable in planar sections, and form a base of various standards and technical norms all over the world up to the present (ASTM, 1996). This intuitive or even confusing development of practical applications is rather curious, because already the above-mentioned Barbier's paper (Barbier, 1860) contained the proposal of an inversion of the games problem allowing for a reliable estimation of certain object properties by counting their point intersections with test probes.

\section{CONCLUSION}

Let us conclude this paper with the words of Florian Cajori (1893): As compared with the vast development of other mathematical branches; the theory of probability has made very insignificant progress since the time of Laplace. ... The only noteworthy recent addition to probability is the subject of "local probability", developed by several English and a few American and French mathematicians. This appraisal suitably characterizes the long way made by geometric probability from the $17^{\text {th }}$ to the beginning of the $20^{\text {th }}$ century. It was thus prepared for its full development in random set theory and vast applications in image and spatial analysis.

\section{ACKNOWLEDGEMENTS}

The authors acknowledge the financial support for this work provided by GAAV, grant IAA801240901, and by the grant MSM 0021620839.

\section{REFERENCES}

Arbuthnot J (1692). Of the laws of chance. London.

ASTM E112 (1996). Standard methods for determining average grain size. ASTM.

Baddeley A, Jensen EBV (2005). Stereology for statisticians. Boca Raton: Chapman\&Hall/CRC.
Barbier J-É (1860). Note sur le problème de l'aiguille et le jeu du joint couvert. J Math Pure Appl 5:273-86.

Barbier J-É (1882). Deux moyens d'avoir $\pi$ au jeu de pile ou face. C R Acad Sci 94:1461-2.

Barbier J-É (1887a). Calcul des probabilités. Généralisation du problème résolu par M. J. Bertrand. C R Acad Sci 105:407.

Barbier J-É (1887b). Théorème relatif au jeu de loto. C R Acad Sci 105:435.

Bat'a J (1927). Some experiments on geometric probabilities [in Czech]. Brno: Masaryk University.

Bertrand J (1864). Traité de calcul différentiel et de calcul intégral, Vol. 1. Paris: Gauthier-Villars.

Bertrand J (1870). Traité de calcul différentiel et de calcul intégral, Vol. 2. Paris: Gauthier-Villars.

Bertrand J (1889). Calcul des probabilités. Paris: GauthierVillars.

Blaschke W (1917). Über affine Geometrie XI: Lösung des 'Vierpunktproblems' von Sylvester aus der Theorie der geometrischen Wahrscheinlichkeiten. Ber Akad Wiss Leipzig 69:436-53.

Blaschke W (1935). Vorlesungen über Integralgeometrie. Leipzig und Berlin: BG Teubner.

Bodziony J, Hübner K (1987). Hugo Steinhaus - an unknown stereologist? Acta Stereol 6/Suppl. II:69-78.

Borel É (1909). Élements de la théorie des probabilités. Paris: Hermann.

Bru B (2006). Les leçons de calcul des probabilités de Joseph Bertrand. J Electron Hist Probab Stat 2-2:1-44.

Buffon G.-L Leclerc de (1777). Essai d'arithmétique morale. Histoire naturelle, générale et particulière, servant de suite à l'Histoire naturelle de l'Homme, Suppl., tome IV. Paris: Imprimerie Royale, 46-148.

Cajori F (1893). A history of mathematics. London: Macmillan, 341-2.

Cartan É (1896). Le principle de dualité et certaines intégrales multiples de l'espace tangentiel et de l'espace réglé. Bull Soc Math Fr 24:140-77.

Cauchy A-L (1832). Mémoire sur la rectification des courbes et la quadrature des surfaces courbes. Paris (lithograph), $11 \mathrm{pp}$ [reprinted in Mem Acad Sci Paris 22(1850):3-15; Oeuvres complètes 1/II, Paris: Gauthier-Villars (1908), 167-77].

Cauchy A-L (1841). Note sur divers théorèmes relatifs à la rectification des courbes, et à la quadrature des surfaces. C R Acad Sci 13:1060-5.

Cavalieri BF (1635). Geometria indivisibilibus continuorum nova quadam ratione promota. Bologna: Clementis Ferronij.

\footnotetext{
${ }^{27}$ For more details about Steinhaus'contribution to stereology see (Bodziony and Hübner, 1987).
} 
Chalkley HW (1943). Methods for quantitative morphological analysis of tissue. $\mathrm{J}$ Natl Cancer Inst 4:47-53.

Cheng SY, Li P, Tian G (1996). A mathematician and his mathematical work. Selected papers of S S Chern. Singapore:Uto-Print.

Chern SS (1942). On integral geometry in Klein spaces. Ann Math (2) 43:178-89. .

Crofton MW (1867a). Note on local probability. Math Questions 7:84-6.

Crofton MW (1867b). Théorème sur une intégrale double définie. C R Acad Sci 65:994-5.

Crofton MW (1868). On the theory of local probability, applied to straight lines drawn at random in a plane; the methods used being also extended to the proof of certain new theorems in the integral calculus. Philos T Roy Soc 158:181-99.

Crofton MW (1885). Probability. Encyclopedia Britannica 19:768-88.

Cruz-Orive LM (1987). Stereology: historical notes and recent evolution. Acta Stereol 6/Suppl. II:43-56.

Czuber E (1884a). Geometrische Wahrscheinlichkeiten und Mittelwerte. Leipzig: Teubner.

Czuber E (1884b). Zur Theorie der geometrischen Wahrscheinlichkeiten. Sitz Akad Wiss Wien 90/II:719_ 42.

Czuber E (1899). Die Entwicklung der Wahrscheinlichkeitstheorie und ihrer Anwendungen. Jahresber DMV 7:1-279.

Czuber E (1903). Wahrscheinlichkeitsrechnung und ihre Anwendungen auf Fehlerausgleichung, Statistik und Lebensversicherung. Leipzig: Teubner [2nd edition: 1908 (vol. 1) and 1910 (vol. 2), 3rd edition: 1914].

Davy P, Miles RE (1977). Sampling theory of opaque spatial specimens. J Royal Stat Soc 39/B:56-65.

Delesse MA (1847a). Procédé mécanique pour détermine la composition des roches. C R Acad Sci 25:544-5.

Delesse MA (1847b). Procédé mécanique pour détermine la composition des roches. Ann Mines 13:379-88.

Deltheil R (1926). Probabilités géometriques. Paris: Gauthier-Villars.

Fontenelle B le B de (1735). Histoire de l'Académie royale des sciences (année 1733). Paris:Imprimerie Royale.

Fréchet M (1921). Remarque sur les probabilités continues. Bull Sc Math (2) 45:87-8.

Fréchet M, Halbwachs M (1924). Calcul des probabilités a la portée de tous. Paris: Dunod.

Glagolev AA (1933). On the geometrical methods of quantitative mineralogical analysis of rocks [in Russian]. Trans Inst Econ Min, Moskva 59:1-47.
Glagolev AA, Gotman ID (1934). An experimental quantitative mineralogical sampling of holes of the Kounrad copper-ore deposit [in Russian]. Trans Inst Econ Min, Moskva 62:1-43.

Godfray H (1866). On some problems in the theory of chances. Math Questions 6:72-4.

Godfray H (1867). At random. Math Questions 7:65-7.

Gouraud Ch (1848). Histoire du calcul des probabilités depuis ses origines jusqu'a nos jours. Paris: A. Durand.

Grattan-Guinness I (1992). A note on The Educational Times and Mathematical Questions. Hist Math 19:76-78.

Hadwiger H (1957). Vorlesungen über Inhalt, Oberfläche und Isoperimetrie. Berlin: Springer Verlag.

Halley E (1693). An estimate of the degrees of mortality of mankind, drawn from curious tables of the births and funerals at the city of Breslaw; with an attempt to ascertain the price of annuities upon lives. Phil Trans 7:596-610.

Havlová V, Mazliak L, Šišma P (2005). Le début des relations mathématiques franco-tchécoslovaques vu à travers la correspondance Hostinský-Fréchet. J Electron Hist Probab Stat 1-1:1-18.

Hostinský B (1917). New solution of Buffon's needle problem [in Czech]. Rozpravy ČAVU 26:8 pp.

Hostinský B (1920). Sur une nouvelle solution du probléme de l'aiguille. B Sci Math 44:126-136.

Hostinský B (1925). Sur les probabilités géométriques. Brno: Masaryk University.

Hostinský B (1926). Geometric probabilities [in Czech]. Prague: JČMF.

Huygens, Ch (1657). De ratiociniis in ludo aleae. In: (Schooten, 1657), 517-534.

Johannsen A (1919). A planimeter method for the determination of the percentage composition of rocks. J Geol 27:276-85.

Joly J (1903). The petrological examination of paving-sets. Proc Roy Dublin Soc 10:62-92.

Kalousová A (2009). Stereology in the 19th century: JosephÉmile Barbier. In: Capasso V et al., eds. Proc 10th Eur Congr Stereol Image Anal. Bologna: Esculapio, 167172.

Klain DA, Rota GC (1997). Introduction to geometric probability. Cambridge: Cambridge Univ Press.

Lämmel R. (1904). Untersuchungen über die Ermittlung von Wahrscheinlichkeiten. Ph.D. thesis, Universität Zürich. Excerpts reprinted in Schneider (1988), 367-377.

Laplace P-S de (1812). Théorie analytique des probabilités. Paris:Imprimerie Royale.

Marinoff L (1994). A resolution of Bertrand's paradox. 
Philos Sci 61:1-24.

Miles R (1972). Multi-dimensional perspectives on stereology. J Microsc 95:181-95.

Miles R (1983). Using 4-linc to estimate $N_{V}$. Acta Stereol 2 (Suppl. I):3-12.

Miles RE, Serra J (eds.). Geometrical Probability and Biological Structures: Buffon's 200th Anniversary. Lect Not Biomath No. 23, Berlin, Heidelberg: Springer.

Moran PAP (1944). Measuring the surface area of a convex body. Ann Math 45:793-9.

Newton I (1967). In: Whiteside DT, ed. The mathematical papers of Isaac Newton. Vol. I. Cambridge: Cambridge Univ Press, 61-2.

Pfiefer RE (1989). The historical development of J. J. Sylvester's four point problem. Math Mag 62:309-17.

Plato J von (1994). Creating modern probability. Its mathematics, physics and philosophy in historical perspective. Cambridge: Cambridge Univ Press.

Poincaré H (1896). Calcul des Probabilités. Paris: G. Carré.

Pólya G (1917). Über geometrische Wahrscheinlichkeiten. Sitzungsber K Akad Wiss Wien 126/IIa:319-328.

Rosiwal AK (1898). Ueber geometrische Gesteinsanalysen. Ein einfacher Weg zur ziffermässigen Feststellung des Quantitätsverhältnisses der Mineralbestandtheile gemengter Gesteine. Verhandlungen KK Geol Reichsanstalt 5-6:143-175.

Saltykov SA (1945). Method of geometric quantitative analysis of metals. USSR patent 72704 [in Russian].

Saltykov SA (1946). The method of intersections in metallography. Zavodskaja Laboratorija 12(9-10):81625 [in Russian].

Saltykov SA (1950). Introduction to stereometric metallography. Yerevan: Acad Sci Armen SSR [in Russian; 2nd and extended edition: Moscow, Metallurgizdat, 1958].

Santaló LA (1976). Integral geometry and geometric probability. Reading: Addison-Wesley.

Schneider I, ed. (1988). Die Entwicklung der Wahrscheinlichkeitstheorie von den Anfängen bis 1933: Einführungen und Texte. Darmstadt: Wiss Buchgesellschaft.

Scheinerman E, Wilf H (1994). The rectilinear crossing number of a complete graph and Sylvester's four point problem of geometric probability. Amer Math Monthly 101:939-43.

Schooten F von (1657). Exercitationum Mathematicarum. Leiden: Johannis Elsevirii.

Shand SJ (1916). A recording micrometer for geometrical analysis. J Geol 24:394-404.

Sheynin OB (1994). Bertrand's work on probability. Arch Hist Exact Sci 48:155-99.
Sheynin OB (2003). Geometric probability and the Bertrand paradox. Historia Sci 13:42-53.

Simpson T (1740). The nature and laws of chance. London: Cave.

Sorby HC (1856). On slaty cleavage, as exhibited in the Devonian limestones of Devonshire. Phil Mag 11:20-37.

Steinhaus H (1930a). Zur Praxis der Rektifikation und zum Längenbegriff. Ber Akad Wiss Leipzig 82:120-30.

Steinhaus H (1930b). Sur la portée pratique et théorique de quelques théorèmes sur la mesure des ensembles de droites. In: Leja F, ed. CR du I Congrès des Mathématiciens des Pays Slaves. Warszawa, 348-54.

Steinhaus H (1931). Lengthmeter (A grid for measuring the length of curves) [in Polish]. Czas Geogr 9:211-5.

Steinhaus H. (1950). Mathematical snapshots. Oxford: Oxford Univ Press.

Sylvester JJ (1864a). Problem 1491. Educ Times 17:20.

Sylvester JJ (1864b). On the real and imaginary roots of algebraical equations. Philos T Roy Soc 154:579-666.

Sylvester JJ (1890). On a funicular solution of Buffon's "problem of the needle" in its most general form. Acta Math 14:185-205.

Thompson WR (1932). The geometric properties of microscopic configurations. I. General aspects of projectometry. Biometrika 24:21-6.

Thompson WR, Hussey R, Matteis JT, Meredith WC, Wilson GC, Tracy FE (1932). The geometric properties of microscopic configurations. II. Incidence and volume of islands of Langerhans in the pancreas of a monkey. Biometrika 24:27-38.

Thomson E (1930). Quantitative microscopic analysis. J Geol 38:193-222 [Russian translation: Mineral'noe syr'e 10:1457-1469].

Todhunter I (1857). Treatise on the integral calculus and its applications with numerous examples. Cambridge, London: Macmillan.

Todhunter I (1865). History of the mathematical theory of probability from the time of Pascal to that of Lagrange. Cambridge, London: Macmillan.

Tomkeieff ST (1945). Linear intercepts, areas and volumes. Nature 155:24.

Tweedie C (1922). James Stirling, a sketch of his life and works along with his scientific correspondence. Oxford: Clarendon Press.

Williamson B (1877). An elementary treatise on the integral calculus. New York: Appleton.

Woolhouse WSB (1866). Problem 1894. Math Questions 5:110-20.

Woolhouse WSB (1867). Some additional observations on the four-point problem. Math Questions 7:81-3. 